\title{
Evaluación de un programa piloto para trabajar habilidades narrativas en niños con hipoacusia usuarios de ayudas auditivas
}

\author{
Pamela Tomicic ${ }^{\text {a,*}}$, Gloria García del Solar a , Isabel Matute a , Javiera Drápela a , Fabiola Marín a , \\ Patricia Castro ${ }^{\text {a }}$ \\ a Facultad de Medicina Clínica Alemana, Universidad del Desarrollo, Chile
}

\section{RESUMEN}

El Discurso Narrativo (DN) es una unidad lingüística compleja utilizada en ciertos contextos y que refleja la organización del pensamiento. La evidencia científica muestra que la población sorda, usuaria de ayudas auditivas, presenta dificultades en los diferentes niveles del lenguaje, tanto expresivos como comprensivos, incluida la habilidad para narrar. Además, existe evidencia de que la intervención terapéutica ayudaría a mejorar su rendimiento. Sin embargo, los datos disponibles sobre las características y abordaje del DN en esta población son escasos. El objetivo del estudio es evaluar un programa piloto para trabajar habilidades narrativas en niños chilenos usuarios de ayudas auditivas. Se estudiaron 22 niños con un promedio de edad de 6,5 años, adaptados con audífonos y/o implante coclear. Se aplicó a este grupo de niños una evaluación inicial del DN utilizando el instrumento Evaluación del Discurso Narrativo (EDNA), obteniéndose la Etapa y Desempeño narrativo de cada niño. Luego, se creó y aplicó individualmente un programa de estimulación del discurso narrativo de 12 sesiones una vez por semana. Finalmente, se repitió la evaluación al final del programa. Se encontraron diferencias significativas entre los resultados obtenidos previo y posterior a la implementación del programa de estimulación. En relación con la Etapa del DN, antes de la intervención el 45,5\% de los niños no estructuraba, lo cual se redujo a un 9.1\% en la evaluación final. En cuanto al Desempeño, previo a la intervención el $72,7 \%$ de los niños presentaba un "déficit narrativo", lo cual se redujo a un 18,2\% posterior a la aplicación del programa.

\section{Evaluation of a pilot program: developing narrative skills in children with hearing aids or cochlear implant}

\section{ABSTRACT}

Narrative discourse is considered a linguistic unit that is used in a specific communicative context, being an indicator of thinking organization. Previous evidence shows how hearing aid users, have difficulties with different language skills, both expressive and comprehensive, including the ability to narrate. Additionally, there is evidence showing how therapeutic intervention would help to improve their narrative performance. However, the information available about the discursive skill and the effect of stimulation programs on it in hearing impaired children is scarce. Accordingly, the present study aims to explore narrative performance in hearing impaired children users of hearing aids/cochlear implants, before and after a narrative speech stimulation program. Twenty-two children diagnosed with bilateral hearing loss users of hearing aids/cochlear implants with a mean age of 6.5 years were included. An initial assessment of the narrative skills was performed using Narrative Discourse Assessment (EDNA), which provided a narrative Stage and a Total score. A twelve-session stimulation program was developed and individually administered to children once a week. Finally, an assessment was performed after the program ended. In the initial assessment, $45.5 \%$ of children did not have a structured narrative speech, a percentage that was reduced to a $9.1 \%$ in the final evaluation. Statistically significant differences were observed on the EDNA scores when comparing initial and final assessments. The results obtained in the present investigation show how children who use hearing aids/cochlear implants improved significantly their narrative abilities after participating in a pilot narrative speech stimulation program.

*Autor/a correspondiente: Pamela Tomicic

Email: pamelatomicic@udd.cl
Palabras clave: Audífonos; Discurso narrativo; Hipoacusia infantil; Implante coclear; Lenguaje; Terapia auditiva

\section{Keywords:}

Hearing aids; Narrative discourse; Childhood hearing impairment; Cochlear implant; Auditory therapy 


\section{INTRODUCCIÓN}

La Hipoacusia Bilateral congénita es una patología que se ha beneficiado de los avances tecnológicos de dispositivos de ayudas auditivas, los que actualmente permiten un total acceso a los sonidos del lenguaje hablado. Este cambio ha permitido incorporar a las personas con discapacidad auditiva a un mundo oral-auditivo (Koop, 2010). Existe consenso en que una temprana adaptación auditiva acorta la brecha de adquisición del desarrollo del habla y del lenguaje entre niños con dificultades auditivas y sus pares normo-oyentes (Crosson \& Geers, 2001; Duchesne Louise \& Marschark Marc, 2019; Ingber \& Eden, 2011; Torres \& Santana, 2001). Sin embargo, a pesar de lo anterior, investigaciones internacionales han demostrado que niños usuarios de ayudas auditivas presentan dificultades en diferentes habilidades lingüísticas (Kretschmer \& Kretschmer, 1994; Moreno-Torres et al., 2011). Principalmente, se ha mostrado que niños usuarios de ayudas auditivas presentan déficit a nivel léxico y sintáctico (Kretschmer \& Kretschmer, 1994).

En las últimas décadas, han surgido estudios mostrando que además de las dificultades a nivel léxico y sintáctico, los niños con dificultades de audición y adaptación auditiva presentan un déficit en el desarrollo de habilidades narrativas (Crosson \& Geers, 2001; Meléndez et al., 2014). Cabe señalar que estas habilidades son un importante predictor del desarrollo del lenguaje oral y escrito, así como del proceso de alfabetización de los niños (Coloma et al., 2012). Dado lo anterior, resulta relevante evaluar el discurso narrativo en esta población.

El discurso narrativo (DN) es entendido como oraciones ordenadas en forma coherente que se relacionan temporal y causalmente en una serie de eventos que se orientan a la resolución de un problema (Bassols \& Torrent, 2012). La evidencia sobre el desarrollo del $\mathrm{DN}$ en niños con dificultades auditivas, aunque escasa, da cuenta de un déficit en el desarrollo narrativo oral de esta población en comparación a la norma (Crosson \& Geers, 2001; Meléndez et al., 2014). Meléndez et al. (2014) evaluaron el desempeño expresivo (presentación, episodios y final) y comprensivo del DN en niños chilenos usuarios de Implante Coclear (IC; $\mathrm{n}=12$ edad= entre 6 y 10 años), utilizando el instrumento de Evaluación del Discurso Narrativo (EDNA). Este estudio mostró que el $42 \%$ de la muestra tuvo un desempeño deficitario en el recontado de cuentos, existiendo ausencia de categorías formales básicas de la superestructura, entendidas como: presentación (personaje y sus atributos, espacio y tiempo en que se encuentra), problema (evento inicial que genera el relato), episodio/s (meta u objetivo que impulsa al personaje, acciones que realiza para alcanzar la meta, obstáculos que dificultan los logros, resultados o consecuencias de los obstáculos) y final (resolución positiva o negativa del problema o evento inicial que generó el relato).

Por su parte, Crosson \& Geers (2001) evaluó niños provenientes de Canadá y Estados Unidos de América, usuarios de IC $(\mathrm{n}=87$, edad promedio $=8$ años y 6 meses). En su estudio demostró que los niños con pobre rendimiento en sus habilidades de percepción acústica del habla, presentaban diferencias estadísticas con los niños normo oyentes en los siguientes aspectos: orientaciones, acciones, complicaciones, evaluación y resolución. De igual forma, Nikolopoulos et al. (2003), en un estudio no controlado realizado en niños provenientes de Reino Unido, usuarios de IC $(n=35$, promedio edad implante $=3$ años y 5 meses), indagó sobre las habilidades discursivas antes de recibir el IC, al año y luego a los dos años. Si bien el estudio logró demostrar un avance estadísticamente significativo a través del tiempo, el rendimiento alcanzado a los dos años fue en promedio inferior a lo esperado para los pares oyentes.

En otro estudio, Boons et al. (2013) compararon las habilidades narrativas de niños provenientes de Flandes y Países Bajos, usuarios de IC, con sus pares oyentes $(\mathrm{n}=66$, edad promedio $=8$ años y 3 meses), los resultados mostraron que los niños con IC tenían competencias similares a sus pares oyentes en cantidad y coherencia, sin embargo, cualitativamente presentaban dificultades en contenido y eficiencia en el recontado de historias.

A pesar de la evidencia sobre las dificultades a nivel de DN presentado por niños con hipoacusia, son escasos los estudios que investigan los beneficios del abordaje terapéutico del DN en las habilidades discursivas de los niños con dificultades auditivas. La evidencia previa en su mayoría está dirigida a la población con dificultades de lenguaje, sin alteraciones auditivas (Zamani et al., 2016). Es relevante destacar que estas intervenciones realizadas en grupos de niños normo oyentes con dificultades en el lenguaje, han demostrado que produce cambios favorables en el DN oral (Acosta et al., 2017; Brown et al., 2014; Maggiolo et al., 2003; Maggiolo et al., 2009; Pavez et al., 2008; Segura, 2016).

En el marco de la población con dificultades auditivas, Ingber \& Eden (2011) realizaron una intervención de 3 meses, una vez por semana de manera individual, en niños usuarios de IC ( $\mathrm{n}=34$ edad $=4$ a 7 años), con el objetivo de mejorar la capacidad de narrar historias. Los niños fueron entrenados para organizar episodios pictóricos de guiones temporales y contar las historias que crearon. Las variables estudiadas fueron dos: organización de secuencias de imágenes y la narración de las imágenes. Los resultados de esta investigación, demostraron una mejora 
significativa en el orden secuencial y en la narración después de la intervención. Otro estudio realizado por Pakulski \& Kaderavek (2012), examinó los efectos de una intervención de lectura didáctica sobre la producción narrativa en niños con hipoacusia ( $n=7$ edad=9 a 11 años). El grupo de estudio (niños con hipoacusia) leyó diferentes cuentos a un compañero de lectura asignado sin hipoacusia, en una de dos condiciones: (1) usando un libro de un determinado estilo narrativo o (2) utilizando un libro de cuentos con manipuladores (es decir, ayuda didáctica basada en pizarra con imágenes). Las lecturas ocurrieron durante cuatro días. Después de las lecturas, se comparó la producción narrativa y la comprensión en las condiciones de "solo lectura" versus "lectura manipuladora" (didáctica). Los datos demostraron que la condición de "lectura + didáctica" generó en una mejor calidad narrativa y de comprensión en el grupo intervenido.

En base a lo expuesto anteriormente y bajo la premisa del déficit lingüístico encontrado en la población de niños con hipoacusia usuarios de ayudas auditivas y la escasa evidencia en el abordaje terapéutico de esta habilidad, se hace necesario realizar mayores estudios que aporten a entender los beneficios de este tipo de intervención. El presente trabajo plantea la pregunta ¿Existe un cambio en el rendimiento discursivo de los niños chilenos hipoacúsicos usuarios de ayudas auditivas después de un programa de estimulación en esta población?

Para responder esta pregunta, la presente investigación tiene como objetivo evaluar un programa de estimulación ("Cuéntame”) enfocado principalmente a las características de la superestructura del DN correspondientes a la Etapa de desarrollo narrativo (Estructura, Transición, No estructura) y Desempeño del mismo (Déficit narrativo, Normal bajo o disminuido, Normal, Muy bueno, Sobresaliente) con el propósito de contribuir a la intervención de esta habilidad lingüística.

\section{MÉTODO}

\section{Diseño}

Este estudio de evaluación de programa piloto presenta un diseño de intervención, cuantitativo de antes y después, no controlado.

\section{Participantes}

\section{Reclutamiento y selección}

Los participantes de este estudio fueron seleccionados por conveniencia dentro de un centro privado de Santiago de Chile. Se eligió este centro porque es uno de los principales lugares de atención de pacientes con hipoacusia (implementados con ayudas auditivas) de la Región Metropolitana.

Para el reclutamiento de los participantes, se realizó una revisión del total de los pacientes del centro del año 2015. Se seleccionaron aquellos niños que cumplían con los siguientes criterios de inclusión: (1) presentar hipoacusia bilateral adaptada con alguna ayuda auditiva (IC y/o audífono), (2) recibir terapia auditiva en el mismo centro al menos una vez por semana, (3) presentar lenguaje expresivo inteligible corroborado mediante evaluación clínica con profesional entrenado, traducido en la posibilidad de transcribir más del $50 \%$ del total de una narración escuchada y por último (4) reconocer del habla en un formato abierto, evidenciado con un $80 \%$ de respuestas correctas en el test Glendonald Auditory Screening Procedure (GASP) (Erber, 1982).

Cabe destacar que las sesiones de terapia auditiva que recibían los niños en el centro, no incluían la estimulación de habilidades discursivas y estaban dirigidas principalmente al desarrollo de las habilidades auditivas para mejorar el lenguaje a través de la audición.

\section{Muestra}

Un total de 22 niños cumplieron con los criterios de inclusión antes mencionados, 9 niños y 13 niñas. Todos ellos fueron invitados a participar de esta investigación, previamente aprobada por el Comité de Ética de la Facultad de Medicina de la Universidad del Desarrollo. Para ello, se contactó a los padres de estos niños con el fin de informar acerca del estudio y solicitar el consentimiento informado, el que debía ser firmado previo a la evaluación. Todos aceptaron participar, por lo que la muestra final correspondió a los 22 niños reclutados en el centro de atención seleccionado.

En la primera evaluación, el promedio de edad de los niños fue de 6,5 años, con un rango de 4 y 14 años, estando todos escolarizados en establecimientos regulares. En cuanto a la implementación auditiva, los niños estaban adaptados con diferentes ayudas auditivas, las que se detallan en la tabla $\mathrm{N}^{\circ} 1$.

Al momento del estudio, todos los menores estaban en la categoría 6 de Geers y Moog (Geers \& Moog, 1990) y todos contaban con implementación auditiva (36,3\% con IC, 36,3\% con audífonos y $27,2 \%$ tanto con IC como audífonos). 
Tabla 1. Tipo de implementación auditiva de los participantes.

\begin{tabular}{lcc}
\hline Categorías & Frecuencia & Porcentaje (\%) \\
\hline Implante coclear unilateral & 5 & 22,7 \\
Audífono unilateral & 3 & 13,6 \\
Ambos (implante coclear + audífono) & 4 & 18,2 \\
Implante bilateral & 2 & 9,1 \\
Audífono bilateral & 5 & 22,7 \\
Implante oído medio unilateral & 1 & 4,5 \\
Implante oído medio y audífono & 1 & 4,5 \\
Implante híbrido y audífono & 1 & 4,5 \\
Total & 22 & 100 \\
\hline
\end{tabular}

\section{Evaluación}

Para la evaluación del desempeño narrativo se utilizó la prueba de Evaluación del Desarrollo Narrativo del niño (EDNA) (Pavez et al., 2008), instrumento estandarizado que permite valorar el rendimiento de las habilidades del discurso narrativo en niños, a través del recontado de cuentos infantiles.

Este instrumento considera tres partes esenciales de la narración (superestructura): Presentación, Episodio y Final. A través de la evaluación de estas partes es posible conocer si el menor es capaz o no de estructurar una narración o si se encuentra en una etapa de transición. Esta prueba establece tres posibles etapas de desarrollo del discurso narrativo.

No estructura narración- Se considera que un niño no estructura una narración cuando se observa alguna de las siguientes situaciones: (1) no cuenta nada, (2) utiliza una o dos oraciones, (3) aglutina secuencias de acciones y/o estados, (4) agrupa enumerativamente en torno a un personaje.

Etapa de Transición - Se considera que un niño se encuentra en esta etapa cuando presenta una de las siguientes combinaciones: (1) una secuencia de acción más un obstáculo, (2) un obstáculo más un resultado o (3) una presentación y episodios (ambos incompletos).

Etapa de estructura - Se considera que el niño se encuentra en esta etapa cuando su narración incluye una de las siguientes combinaciones: (1) presentación y episodio (pero solo uno de ellos completo), (2) presentación completa más episodio completo (pero sin final) o (3) las narraciones presentan presentación completa, episodio completo y final.
Las puntuaciones correspondientes a los diferentes desempeños aparecen en la pauta de cotejo y a través del análisis de los recontados, se asigna un puntaje total, el cual permite establecer la categoría del desempeño narrativo.

\section{Instrumentos}

\section{Intervención}

Para la intervención, se creó un programa de estimulación del DN llamado "Cuéntame". El diseño de esta intervención está basado en el plan de estimulación del desarrollo narrativo EDEN, planteado por las mismas autoras del test EDNA.

El plan EDEN es una propuesta teórica-práctica, que se ordena en relación a las etapas del desarrollo narrativo, estructurándose en tres programas; Programa I: Los guiones, Programa II: La estructura narrativa y Programa III: Narraciones más complejas y relato de experiencias personales. La intervención diseñada para este estudio siguió el orden del programa propuesto por EDEN y algunos aspectos de sus unidades.

El programa "Cuéntame" consiste en 12 sesiones de trabajo progresivas en complejidad el cual fue realizado con una frecuencia de una vez por semana. Este programa fue diseñado para ser utilizado en todos los sujetos del estudio, independiente del nivel inicial del DN de cada uno.

Cada sesión del programa cuenta con un objetivo y una actividad para cumplir dicho objetivo, comenzando con el trabajo de guiones, luego la presentación del cuento, episodios y final. Cada una posee además una "sesión extra", que tiene el mismo objetivo y similar actividad que la sesión original, la que se realiza en la siguiente sesión, solo en el caso que no se haya alcanzado el objetivo declarado.

Las sesiones de trabajo cuentan con un set de material específico, que incluye láminas y objetos necesarios para realizar las actividades y cumplir los objetivos planteados.

La sesión 1 y 2 se centra en el recontado de guiones a través del uso de láminas, la sesión 3 aborda la narración de acciones en torno a un personaje utilizando una secuencia de imágenes, para la sesión 4 se utiliza un objeto concreto (perro de peluche) para trabajar la categoría formal de presentación, para la sesión 5 y 6 se abordan las categorías de presentación y episodio completo, usando tanto láminas como objetos concretos, desde la sesión 7 hasta la 12 se abordan todas las categorías formales del cuento, es decir: presentación, episodios y final, el aumento de la complejidad está dado por la retirada progresiva de los apoyos visuales y la cantidad de episodios de cada cuento. 


\section{Procedimiento}

El estudio consideró tres etapas: la primera, una evaluación inicial del DN (pre-intervención), luego la aplicación del programa de estimulación (intervención) y finalmente una evaluación final (post-intervención inmediata).

Para llevar a cabo esta investigación, se contactó a las educadoras y fonoaudiólogas a cargo de las terapias de los niños en el centro donde ellos eran atendidos y se les invitó a ser parte de esta investigación. Ellas serían las encargadas de evaluar y aplicar el programa. Todas las profesionales a cargo de los niños aceptaron participar. Para cautelar tanto la fidelidad de la intervención como las evaluaciones, se realizó una jornada de 8 horas de capacitación en relación al instrumento de evaluación a utilizar en el estudio (protocolo de evaluación EDNA) y sobre el programa de estimulación que debían implementar. Se entregó además, un instructivo explicativo del material de estimulación que recibirían y el detalle de las actividades para cada una de las sesiones. Una de las investigadoras estuvo a cargo de la supervisión y seguimiento de las terapias realizadas, asistiendo a las sesiones de intervención (de manera aleatoria) y resolviendo dudas vía telefónica o de manera presencial.

Finalizado el proceso, se realizó una jornada de cierre con las educadoras del centro, donde se mostraron los principales resultados del estudio obtenido y a cada profesor se le entregó como agradecimiento un libro de estimulación del discurso narrativo (EDNA) de las autoras Pavez et al. (2008).

El 100\% de los niños asistió a las sesiones de intervención individual, una vez a la semana, las cuales fueron realizadas en las sesiones habituales de terapia de cada uno, entre marzo y noviembre del 2015. Para las evaluaciones inicial y final, se destinó una sesión completa de $45 \mathrm{~min}$. y para la aplicación del programa de estimulación se destinaron $15 \mathrm{~min}$. aproximadamente en cada sesión.

Durante la evaluación, las producciones orales de los recontados de los cuentos, fueron grabadas en audio y transcritas por las investigadoras. Con el fin de asegurar la confiabilidad de la transcripción, se definió el mismo procedimiento para todas las grabaciones, estas fueron; transcribir al pie de la letra y palabra por palabra, manteniendo las repeticiones frecuentes y anotando las pausas.

El análisis de los corpus, lo realizó una sola investigadora con el fin de evitar diferencias de criterio, quien se ajustó a los parámetros indicados por el test EDNA.

\section{Análisis de datos}

Los datos recogidos fueron analizados con el programa estadístico SPSS 22. La etapa y desempeño del DN fueron analizadas de manera descriptiva (utilizando frecuencia y porcentaje) y luego se trabajó comparando las proporciones de los niños en las distintas categorías en las dos mediciones (pre y post intervención). Se utilizó la prueba no paramétrica de comparación de proporciones para dos muestras relacionadas, McNemar-Bowker, con el objetivo de constatar el cambio y así determinar que la diferencia no se deba al azar.

Al no observar diferencias en los resultados de los niños en relación a las co-variables estudiadas, se descartaron análisis multivariados. Para todos los análisis se consideró un nivel de significancia del $\mathrm{p}<0,05$.

Las variables analizadas fueron:

A. Etapa del DN: Esta es una variable cualitativa ordinal de tres categorías: No estructura, Transición, estructura.

B. Desempeño narrativo: Esta es una variable continua en función de percentiles $(0-100)$, que luego se re-categoriza en 5 categorías ordinales:

1. Déficit narrativo: igual o menor a percentil 10 .

2. Normal bajo o disminuido: entre los percentiles 10 e inferior a 25 .

3. Normal: entre los percentiles 25 y 75.

4. Muy bueno: igual o superior al percentil 75 .

5. Sobresaliente: superior al percentil 90.

Para efectos de análisis, esta variable fue dicotomizada en: "Déficit o Normal bajo" y "Normal o mejor".

\section{RESULTADOS}

Los aspectos estimulados en el programa "Cuéntame" apuntaron a la mejora de la etapa del DN y al desempeño alcanzado. En todos estos aspectos se evidenciaron progresos significativos en los niños estudiados.

\section{Resultados variable Etapa del DN}

En relación a la etapa del DN, se observó que posterior a la intervención, la mayoría de los niños avanzaron a la etapa 
estructura $(72,7 \%)$ y solo un grupo menor se ubicó en la etapa no estructura $(9,1 \%)$ (tabla $\left.\mathrm{N}^{\circ} 2\right)$.

Tabla 2. Evaluación pre y post intervención según la etapa del DN.

\begin{tabular}{llcccc}
\hline \multirow{2}{*}{ Variables } & \multicolumn{2}{c}{ Primera evaluación } & \multicolumn{2}{c}{ Segunda evaluación } \\
\cline { 3 - 6 } & & $\mathrm{N}$ & $\%$ & $\mathrm{~N}$ & $\%$ \\
\hline \multirow{3}{*}{ etapa } & Estructura & 8 & 36,4 & 16 & 72,7 \\
& Transicion & 4 & 18,2 & 4 & 18,2 \\
& No estructura & 10 & 45,5 & 2 & 9,1 \\
\hline
\end{tabular}

$\mathrm{N}$ : número de niños. \%: porcentaje de niños, ubicados en las distintas etapas del $\mathrm{DN}$, para la primera y segunda evaluación.

Al comparar las etapas en que se encontraban los niños en las dos evaluaciones, se observan diferencias significativas en los cambios de una etapa a otra con un valor $\mathrm{p}=0,012$ (McNemarBowker).

\section{Evolución de la variable Etapa del DN}

Observando los resultados totales de las etapas del discurso narrativo de cada niño, se refleja un cambio favorable. La mayoría $(85,7 \%)$ de los niños avanzaron de etapa de estructuración, luego de participar en el programa de estimulación. Para un análisis más detallado, a continuación se presenta cuántos menores se mantienen o avanzan desde su categoría según su primera evaluación.

Como se observa en la tabla 2 previo a la aplicación del programa 10 de los menores evaluados no estructuraban una narración. Cabe señalar que todos ellos lograban narraciones aglutinando secuencias de acciones y/o estados o agrupaban enumerativamente en torno al personaje. Posterior a la intervención se observó que 5 de estos menores avanzaron a la etapa de estructura, 3 avanzaron a la etapa de transición y 2 se mantuvieron en la misma etapa. De los 5 menores que avanzaron a la etapa de estructura, se observó que 3 de ellos lograron narraciones con presentación y episodio (sólo uno de ellos completo), los otros 2 menores realizaron presentación y episodio completos, pero sin final. De los 3 menores que avanzaron a la categoría transición, 2 de ellos realizaron narraciones con una secuencia de acción más obstáculo y para 1 de ellos se observó una presentación con un episodio (ambos incompletos). Para los 2 menores que mantuvieron su categoría, es posible advertir que solo uno de ellos mejora su nivel de narración, sin embrago, esto no le permite movilizarce a la siguiente categoría.

Para el caso de los 4 menores que se encontraban en la categoría de transición previo a la intervención, se observó que 3 de ellos mostraban narraciones con presentación y episodios (ambos incompletos) y 1 de ellos presentaba un nivel inferior que incluía una secuencia más obstáculo, posterior a la intervención se observó que 3 avanzaron a la etapa estructura, y solo 1 se mantuvo en su categoría de transición.

De los 3 menores que avanzaron a la categoría estructura, se observaron tres rendimientos diferentes: uno de ellos logró una presentación incompleta y episodio completo, otro obtuvo una presentación y episodio completos pero sin final y el tercero realizó una presentación completa, un episodio completo y final. No se observaron cambios en el menor que mantuvo en transición.

En relación a los 8 niños que se mantuvieron en su categoría estructura, también fue posible apreciar mejoras en sus narraciones. Previo al programa 4 menores de este grupo, eran capaces de realizar narraciones con una presentación completa y un episodio incompleto, 2 de ellos lograban una presentación completa más un episodio completo y 2 de ellos lograban incorporar el final. Para la evaluación post intervención, se apreció en 5 menores la incorporación de elementos como: presentación completa, episodio completo y final. Para los otros 3 menores se apreció un rendimiento similar pero sin final.

\section{Variable Desempeño del DN}

En relación con el desempeño narrativo de los niños, se observó que previo a la aplicación del programa la mayoría de los niños presentaba un "déficit narrativo" (72,7\%). Posterior a la intervención, la mayoría de los niños avanzaron a la categoría "normal" (54,5\%) o mejor (tabla $\mathrm{N}^{\circ} 3$ ).

Tabla 3. Evaluación pre y post intervención según la etapa del DN.

\begin{tabular}{llcccc}
\hline Variables & & \multicolumn{2}{c}{$\begin{array}{c}\text { Primera } \\
\text { evaluación }\end{array}$} & \multicolumn{2}{c}{$\begin{array}{c}\text { Segunda } \\
\text { evaluación }\end{array}$} \\
\cline { 3 - 6 } & & $\mathrm{N}$ & $\%$ & $\mathrm{~N}$ & $\%$ \\
\hline \multirow{3}{*}{ Desempeño } & Deficit narrativo & 16 & 72,7 & 4 & 18,2 \\
& Normal bajo & 3 & 13,6 & 3 & 13,6 \\
& Normal & 3 & 13,6 & 12 & 54,5 \\
& Muy bueno & 0 & 0 & 2 & 9,1 \\
& Sobresaliente & 0 & 0 & 1 & 4,5 \\
\hline
\end{tabular}

$\mathrm{N}$ : número de niños; \%: porcentaje de niños, ubicados en las distintas categorías de desempeño, para la primera y segunda evaluación. 
Para efectos del análisis, esta clasificación del desempeño narrativo fue dicotomizada en: "Déficit o Normal bajo" y "Normal o mejor". Se compararon estos resultados tomando en cuenta la primera y la segunda evaluación y se encontraron diferencias estadísticamente significativas $(\mathrm{p}=0,002)$ (McNemarBowker).

Usando la dicotomización de sus categorías (agrupación en dos categorías: "déficit o normal bajo" y "normal o mejor"), los resultados totales también mostraron un cambio favorable en la variable de desempeño. Para los 18 niños que se ubicaban en la categoría de déficit narrativo o normal bajo, 12 de ellos mejoraron y 6 se mantuvieron en su categoría. En el caso de los 4 niños que se ubicaban en la categoría de normal o mejor, solo uno de ellos empeoró su desempeño ubicándose en la categoría de déficit narrativo o normal bajo.

Al igual que en la etapa, es posible evidenciar una mejoría en el desempeño del DN en la mayoría de los niños $(66,6 \%)$ luego de participar en el programa de estimulación.

\section{DISCUSIÓN}

Los resultados obtenidos en la presente investigación muestran que el programa de intervención piloto "Cuéntame", mejoró de manera importante las habilidades narrativas de los niños usuarios de ayudas auditivas. Ello muestra la relevancia de abordar de manera planificada este tipo de contenidos, considerados relevantes, entre otras cosas, para el desempeño escolar.

Es importante observar el rendimiento obtenido en la evaluación pre-intervención, la que deja en evidencia un pobre desempeño del DN en esta población. Si bien todos los niños contaban con excelentes habilidades de escucha (Categoría 6 de Geers y Moog), 10 niños de un total de 22, se ubicaron en la etapa de no estructura tomando en consideración que, según la edad cronológica (mayor a 4 años), todos ellos debieran estar en la etapa de estructurar el discurso. Por otra parte, a nivel de desempeño narrativo, la mayoría de los niños (16 niños de un total de 22) obtuvo un rendimiento igual o menor al percentil 10 , correspondiente a la categoría "déficit narrativo", que es el nivel más bajo de desempeño narrativo. Estos resultados son concordantes con los estudios previos que muestran un déficit del DN en esta población (Crosson \& Geers, 2001; Meléndez et al., 2014; Nikolopoulos et al., 2003).

Es posible hacer algunas reflexiones sobre el bajo rendimiento del DN en los niños usuarios de dispositivos de ayudas auditivas.
Existe vasta evidencia que considera que el desarrollo del lenguaje es directamente dependiente de la calidad perceptiva o input recibido. Sin embargo, aunque el niño sordo adaptado con ayudas auditivas posea un input "adecuado", esto no es suficiente para normalizar habilidades narrativas en esta población. Ello muestra la necesidad de un abordaje específico de esta habilidad. A este respecto es necesario destacar que un adecuado input permite contar con una base adecuada para poder estimular el desarrollo de habilidades narrativas, Así lo demostraron Crosson \& Geers (2001) donde los niños con mejor percepción del habla consiguieron mejores avances tanto en estructura como cohesión del DN.

En línea con evidencia previa, los niños evaluados en este estudio presentaron un pobre rendimiento a nivel del DN a pesar de que todos ellos contaban con la habilidad de percibir el habla en un formato abierto, es decir sin contar con pistas visuales (categoría 6 de Geers y Moog), lo que habitualmente se relaciona directamente con mejores habilidades lingüísticas. Esto se puede explicar dado que el DN es un aspecto del desarrollo del lenguaje que necesita que el menor sea capaz de ordenar de manera coherente eventos que se dirigen a la resolución de un problema lo que involucra habilidades más allá del solo reconocimiento de palabras en un formato abierto.

Los dispositivos de ayuda auditiva restauran la audición en los niños, sin embargo, existen factores propios del niño y del ambiente que pueden incidir negativamente en el desarrollo de las habilidades narrativas, por ejemplo: retraso de lenguaje, etiología de la pérdida, edad de implantación, educación de los padres, dificultad para escuchar en ambientes ruidosos, etc. (Huttunen, 2008; Murri et al., 2015; Spencer et al., 2003). Estas condiciones, sumado a la evidencia del déficit narrativo, fortalecen la idea de que, además de la adaptación auditiva y la estimulación del desarrollo de las habilidades auditivas, es necesario intervenir las habilidades discursivas en esta población.

La aplicación del programa "Cuéntame" apuntó a la mejora de la superestructura narrativa (presentación, episodios, final), específicamente a la etapa del discurso narrativo y al nivel de desempeño. Para la etapa del discurso narrativo, de los 14 niños que no lograron estructurar el discurso, solo 2 del total de la muestra no logró la etapa "estructura" al final de la intervención. Esto significa que la mayoría de los niños logró un avance en la estructuración formal de los cuentos.

Este avance se demuestra en la incorporación de las categorías básicas de la superestructura: en la presentación del cuento se observó la incorporación del personaje y el problema que 
desencadena la historia, así como en los episodios se evidenció la incorporación de acciones, obstáculos y resultados en sus narraciones.

Por otra parte, de los 16 niños de la muestra que se encontraban en déficit, solo 4 de ellos quedaron en esta categoría, demostrando el progreso en el desarrollo narrativo en relación a su grupo de edad, esto debido a que incorporaron elementos de la superestructura que aumentaron su puntuación total, ubicándolos en una mejor categoría.

Estos resultados son relevantes y coinciden con otros estudios sobre la utilidad de intervenir a través de programas estructurados estimulación del DN (Ingber \& Eden, 2011). Cabe destacar que la presente investigación consideró una población que ha sido poco abordada anteriormente en este tipo de intervenciones, evidenciado a través de búsquedas bibliográficas en bases de datos compiladas. Por lo anterior, este estudio piloto constituye una evidencia pionera en Chile, en relación a la descripción de habilidades narrativas en niños sordos usuarios de ayudas auditivas y sugiere la importancia de continuar la investigación en esta línea.

De todas maneras, es importante mencionar que el presente estudio posee limitaciones. En primer lugar, el tamaño de la muestra no permite generalizar estos resultados en una población mayor, sin embargo, al ser un estudio piloto entrega datos suficientes para realizar en el futuro un estudio con un mayor número de participantes. Otra limitación del estudio es la ausencia de un grupo control, el cual permitiría evaluar de manera más directa si la mejora de las habilidades son parte de los avances propios del desarrollo o producto de la intervención realizada. Pese a lo anterior, la evidencia en el progreso de las habilidades narrativas posterior a la aplicación del programa "Cuéntame" establece bases para conocer cómo intervenir de manera eficiente en esta población caracterizada por presentar dificultades en estas habilidades lingüísticas complejas.

Otra reserva del estudio fue no considerar otros factores lingüísticos como los comprensivos/expresivos, fonológicos, semánticos y morfosintácticos, que podrían interferir en las producciones orales de los recontados. Junto a esto, seria relevante incluir otros antecedentes auditivos, además de la edad de implementación del dispositivo de ayuda auditiva. También es necesario considerar para futuras investigaciones un método objetivo para cautelar la confiabilidad de las transcripciones y el análisis de los corpus, y de esta manera disminuir el efecto del azar en la proporción de la concordancia observada. A pesar de lo anterior, el estudio se ciñó a las directrices para la corrección entregadas por los autores del EDNA, siendo un solo evaluador el encargado de este análisis, con la finalidad de evitar diferentes criterios para las transcripciones.

En síntesis, esta investigación muestra que los niños con dificultades auditivas presentan problemas a nivel del DN a pesar de contar con un adecuado nivel de percepción del habla. Conjuntamente, los resultados de este estudio muestran que un programa estructurado dirigido al abordaje de las dificultades narrativas tiene un impacto positivo en el desempeño narrativo de los niños con dificultades de audición. Ello evidencia la necesidad de investigar las habilidades discursivas en los niños sordos usuarios de ayudas auditivas, dado la presencia de dificultades a este nivel en la población sorda y la importancia de estas habilidades como predictoras del desarrollo del lenguaje oral y escrito. Además, muestra el beneficio de contar con programas de intervención dirigidos específicamente a abordar el DN en la población con dificultades auditivas. Al respecto, es importante plantear que se requiere más evidencia científica sobre los mejores métodos de evaluación e intervención del DN en esta población. Ello con el objetivo de mejorar el abordaje de los aspectos deficitarios en esta población.

\section{REFERENCIAS}

Acosta, V., Moreno, A. M., \& Axpe, M. Á. (2017). La detección e intervención en habilidades narrativas en niños con trastorno específico del lenguaje en contextos $\begin{array}{llll}\text { educativos. } & \text { Educación } & \text { XXI, } & \text { 20(2), }\end{array}$ https://doi.org/10.5944/educxx1.19053

Bassols, M., \& Torrent, A. M. (2012). Modelos textuales: Teoria y practica. Octaedro.

Boons, T., De Raeve, L., Langereis, M., Peeraer, L., Wouters, J., \& van Wieringen, A. (2013). Narrative spoken language skills in severely hearing impaired schoolaged children with cochlear implants. Research in Developmental Disabilities, 34(11), 3833-3846. https://doi.org/10.1016/j.ridd.2013.07.033

Brown, J. A., Garzarek, J. E., \& Donegan, K. L. (2014). Effects of a Narrative Intervention on Story Retelling in At-Risk Young Children: Topics in Early Childhood Special Education. https://doi.org/10.1177/0271121414536447

Coloma, C. J., Pavez, M. M., Peñaloza, C., Araya, C., Maggiolo, M., \& Palma, S. (2012). Desempeño lector y narrativo en escolares con trastorno específico del lenguaje. Onomázein, 26(2), 351-375. http://www.redalyc.org/articulo.oa?id=134525391013

Crosson, J., \& Geers, A. (2001). Analysis of narrative ability in children with cochlear implants. Ear and Hearing, 22(5), 381-394. https://doi.org/10.1097/00003446-200110000-00003

Duchesne Louise, \& Marschark Marc. (2019). Effects of Age at Cochlear Implantation on Vocabulary and Grammar: A Review of the Evidence. American Journal of Speech-Language Pathology, 28(4), 1673-1691. https://doi.org/10.1044/2019_AJSLP-18-0161 
Erber, N. P. (1982). Auditory training. Alexander Graham Bell Association for the Deaf. http://www.agbell.org/

Huttunen, K. (2008). Development of speech intelligibility and narrative abilities and their interrelationship three and five years after paediatric cochlear implantation. International Journal of Audiology, 47 Suppl 2, S38-46. https://doi.org/10.1080/14992020802322619

Ingber, S., \& Eden, S. (2011). Enhancing sequential time perception and storytelling ability of deaf and hard of hearing children. American Annals of the Deaf, 156(4), 391-401. https://doi.org/10.1353/aad.2011.0033

Koop, C. E. (2010). Foreword: Pursuing Excellence in Early Hearing Detection and Intervention Programs. Pediatrics, 126(Supplement 1), S1-S2. https://doi.org/10.1542/peds.2010-0354C

Kretschmer, R., \& Kretschmer, L. (1994). Discourse and hearing-impairment. En D. Ripich \& N. A. Creaghead (Eds.), School Discourse Problems (pp. 349-378). Singular Publishing.

Maggiolo, M., Coloma, C. J., \& Pavez, M. M. (2009). Estimulación de narraciones infantiles. Revista CEFAC, 11(3), 379-388. https://doi.org/10.1590/S151618462009005000043

Maggiolo, M, Pavez, M. M., \& Coloma, C. J. (2003). Terapia para el desarrollo narrativo en niños con trastorno específico del lenguaje. Revista de Logopedia, Foniatría y Audiología, 23(2), 98-108. https://doi.org/10.1016/S02144603(03)75750-7

Meléndez, J., Valdebenito, J., \& Meza, P. (2014). Desempeño narrativo en un grupo de 6 a 10 años con Implante coclear unilateral en categoría 6 de escala Geers y Moog pertenecientes a un centro privado (tesis de pregrado). Universidad del Desarrollo.

Moreno-Torres, I., Cid, M. del M., Santana, R., \& Ramos, Á. (2011). Estimulación temprana y desarrollo lingüístico en niños sordos con implante coclear: El primer año de experiencia auditiva. Revista de Investigación en Logopedia, 1(1), 56-75. https://revistas.ucm.es/index.php/RLOG/article/view/58707

Murri, A., Cuda, D., Guerzoni, L., \& Fabrizi, E. (2015). Narrative abilities in early implanted children. The Laryngoscope, 125(7), 1685-1690. https://doi.org/10.1002/lary.25084

Nikolopoulos, T. P., Lloyd, H., Starczewski, H., \& Gallaway, C. (2003). Using SNAP Dragons to monitor narrative abilities in young deaf children following cochlear implantation. International Journal of Pediatric Otorhinolaryngology, 67(5), 535-541. https://doi.org/10.1016/s0165-5876(03)00034-x

Pakulski, L. A., \& Kaderavek, J. N. (2012). Reading Intervention to Improve Narrative Production, Narrative Comprehension, and Motivation and Interest of Children with Hearing Loss. Volta Review, 112(2), 87-112.

Pavez, M. M., Maggiolo, M., \& Coloma, C. J. (2008). Test para evaluar procesos de simplificación fonológica: TEPROSIF-R. Ediciones UC.

Segura, H. (2016). Eficacia temprana de un programa de intervención del discurso narrativo para niños con Trastorno Específico del Lenguaje (TEL). Revista Digital EOS, 7(1), 14-26.

Spencer, L. J., Barker, B. A., \& Tomblin, J. B. (2003). Exploring the Language and Literacy Outcomes of Pediatric Cochlear Implant Users. Ear and hearing, 24(3), 236-247. https://doi.org/10.1097/01.AUD.0000069231.72244.94

Torres, S., \& Santana, R. (2001). Intervención logopédica con sordos: Procedimientos, tareas y materiales. En M. Puyuelo (Ed.), Intervención Del Lenguaje: Metodología y Recursos Educativos: Aplicaciones Especificas a la Deficiencia Auditiva (pp. 49-79). Masson.

Zamani, P., Soleymani, Z., Khatoonabadi, A. R., Rezaei, M., \& Rezai, H. (2016). A systematic review of narrative-based language intervention with children who have language disorders caused by hearing impairments. Auditory and Vestibular Research, 25(2), 98-110. https://avr.tums.ac.ir/index.php/avr/article/view/96 